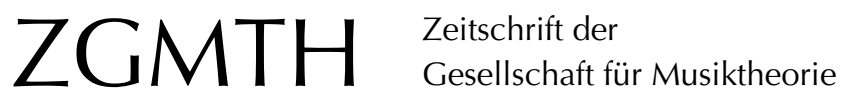

Sprick, Jan Philipp (2006): Historie versus Systematik? - Die Kongresse der GMTH und SMT in Hamburg und Boston im Herbst 2005. ZGMTH 3/1, 153-163.

https://doi.org/10.31751/219

(C) 2006 Jan Philipp Sprick

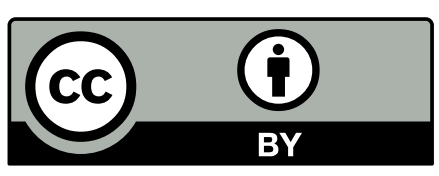

Dieser Text erscheint im Open Access und ist lizenziert unter einer Creative Commons Namensnennung 4.0 International Lizenz.

This is an open access article licensed under a

Creative Commons Attribution 4.0 International License.

veröffentlicht / first published: 01/01/2006

zuletzt geändert / last updated: 01/12/2008 


\section{Historie versus Systematik? Die Kongresse der GMTH und SMT in Hamburg und Boston im Herbst 2005}

Die bereits seit Mitte der 60er Jahre akademisch institutionalisierte `Music Theory der USA ist ein wichtiges Vorbild für die im Aufbruch begriffene Musiktheorie Europas. ${ }^{1}$ Gleichwohl sind europäische und amerikanische Musiktheorie in inhaltlicher wie institutioneller Hinsicht zwei deutlich unterschiedliche Diziplinen. Wird auch beiden gleichermaßen abverlangt, in der Lehre einen Hauptakzent auf die propädeutische Pflichtfachausbildung zu legen, kann doch die sMusic Theorys in den USA auf eine lange und eigenständige Forschungstradition zurückblicken und erscheint, da sie zumeist in die Music Departments der Universitäten integriert ist, als akademische Disziplin institutionell gefestigt. Demgegenüber befindet sich zumal die Musiktheorie in Deutschland in einer prekären Situation: Institutionell an den Musikhochschulen verankert, nimmt sich ihre Möglichkeit, einen identitätsstiftenden Rückhalt in der eigenen wissenschaftlichen Ausrichtung zu finden, noch vergleichsweise bescheiden aus.

Eindrücke vom Hamburger Kongress der Gesellschaft für Musiktheorie und dem Annual Meeting der Society for Music Theory (SMT) in Boston seien im Folgenden die Grundlage für einige Überlegungen zum gegenwärtigen Stand der deutschen und amerikanischen Musiktheorie. Dabei wird sich zeigen, dass das durch das Motto des Dresdener Gründungskongresses der GMTH im Jahr 2001 »Musiktheorie - Zwischen Historie und Systematik« beschriebene Spannungsfeld eine für die Disziplin unverminderte Aktualität besitzt.

\section{»Musiktheorie im Kontext»}

Auch im fünften Jahr nach Gründung der GMTH bilden deren Jahreskongresse einen Gradmesser für die inhaltliche Entwicklung der Musiktheorie in Deutschland. Jahrestreffen akademischer Organisationen haben - anders als Fachkongresse - in erster Linie zum Ziel, einen möglichst breiten Überblick über aktuelle Diskussionen innerhalb der eigenen Disziplin zu geben. Gleichzeitig sind sie ein wichtiges Forum für den intensiven fachlichen und persönlichen Austausch ihrer Mitglieder. Der V. Kongress der GMTH, der vom 14. bis 16. Oktober 2005 an der Hochschule für Musik und Theater in Hamburg stattfand, stand unter dem Motto »Musiktheorie im Kontext«. Über 200 offiziell registrierte Teilnehmer waren an die Hamburger Musikhochschule gekommen. Unter den passiven wie aktiven Teilnehmern befand sich eine auffallend hohe Zahl jüngerer Lehrbeauftragter. Sie repräsentieren eine Gruppe, die bei den Treffen der GMTH nicht zuletzt

1 Ein Indiz des Aufbruchs sind die zahlreichen Neugründungen nationaler Gesellschaften für Musiktheorie: In Italien wurden 1989 gleich zwei Gesellschaften für Musiktheorie gegründet. Es folgten Gründungen in Frankreich, Spanien, Belgien und England. Als letzte Gründung vor derjenigen der GMTH (2000) erfolgte 1999 die der Dutch Society for Music Theory in den Niederlanden. 
auch die Erfahrung professioneller Zugehörigkeit sucht, die ihnen infolge des ausgesprochen sinformellen Status der Lehrbeauftragten hierzulande an der eigenen Hochschule oft verwehrt bleibt.

Wie seine Vorgänger fand auch der Hamburger Kongress erneut unter großer internationaler Beteiligung statt, wobei insbesondere die Präsenz einiger der führenden Vertreter aktueller Strömungen der amerikanischen Musiktheorie hervorzuheben ist. Dies ist ein Zeichen dafür, dass sich die Kongresse der GMTH innerhalb nur weniger Jahre zu einem international beachteten Forum der Musiktheorie entwickelt haben. Dies lässt auf eine zukünftige produktive Beziehung von amerikanischer und deutscher Musiktheorie hoffen. Denn obwohl die deutsche Musiktheorie seit ihrer Institutionalisierung durch die GMTH wieder stärker von der amerikanischen Musiktheorie wahrgenommen wird, ist die aktive Rezeption deutschsprachiger musiktheoretischer Literatur in den USA noch immer beklagenswert gering. Ein fachlicher Dialog findet nach wie vor primär auf der Grundlage persönlicher Kontakte statt, die allerdings gerade von amerikanischen Musiktheoretikerinnen und -theoretikern zunehmend gesucht werden. Zu dieser Entwicklung passt, dass sich unter den eingereichten Abstracts eine erstaunlich hohe Zahl von Bewerbungen aus den USA befand, die nicht nur - und das sei eigens betont - auf persönliche Aufforderung hin, sondern zum großen Teil durch die Verbreitung des Call for Papers über die Mailingliste der SMT zustande gekommen war. ${ }^{2}$

Ohnehin war mit 100 Einsendungen die Zahl der Proposals so hoch wie noch nie. Die Qualität der Beiträge hatte die Vorbereitungsgruppe des Kongresses ${ }^{3}$ und den Vorstand der GMTH dazu bewogen, die vorgesehene Zahl der Vorträge von 40 auf 60 zu erhöhen. Der Überlegung folgend, dass »durch die ästhetische Praxis, benachbarte Disziplinen und den geschichtlichen Hintergrund der Musiktheorie eine Reihe von Fragestellungen erwachsen, die über die Grenzen eines propädeutisch verengten Fachverständnisses hinausweisen ${ }^{4}$, bildete eine interdisziplinäre Ausrichtung die zentrale inhaltliche Akzentuierung der Veranstaltung. Besonders zum Ausdruck kam dies in der von Christian Thorau (Frankfurt a. M.) inhaltlich betreuten Sektion »Zeichentheoretische und kognitivistische Ansätze in der Musiktheorie«. Weitere thematische Sektionen widmeten sich der Wechselwirkung von Musiktheorie und ästhetischer Erfahrung, dem Verhältnis von Musiktheorie und Komposition sowie den Bezügen zwischen Musiktheorie und kulturellem Kontext.

Die hohe Anzahl der Vorträge und das breit gefächerte thematische Spektrum des Hamburger Kongresses erlauben keine detaillierte Beschreibung seines Verlaufs. Die folgenden Ausführungen - und gleiches gilt für die Darstellung des amerikanischen Kongresses - geben zwangsläufig einen ausschnittartigen und subjektiven Eindruck wieder.

Jeder der vier thematischen Sektionen war ein Einführungsvortrag vorangestellt: Als Auftakt der Sektion »Musiktheorie und ästhetische Erfahrung« entwickelte Elmar Lamp-

2 Gerade vor diesem Hintergrund wäre es wünschenswert, dass sich auch deutsche Musiktheoretikerinnen und -theoretiker verstärkt mit eigenen Vorschlägen für die Teilnahme an SMT-Meetings in den USA bewerben.

3 Mitglieder der Vorbereitungsgruppe waren: Reinhard Bahr (Kongressleitung), Jan Philipp Sprick, Catherine Fourcassie, Jörn Arnecke, Wolfgang-Andreas Schultz, Axel Schaffran.

4 Textlaut des Call for papers. 
son, Präsident der Hamburger Musikhochschule, in seinem Referat »Musiktheorie als intelligentes Fühlen« Perspektiven einer stärker an der musikalischen Erfahrung orientierten Musiktheorie. Die ursprünglich im Hinblick auf Fragestellungen der praktischen Pädagogik hin konzipierte Sektion erwies sich schließlich als die inhaltlich heterogenste. Das Spektrum der zwölf Beiträge reichte von primär philosophisch orientierten Vorträgen wie denjenigen von Jörg-Peter Mittmann (»Überlegungen zum empirischen Wert musiktheoretischer Aussagen «) und Martin Kaltenecker (»Zum Begriff der musikalischen Zuschreibung ") bis hin zu pädagogisch ausgerichteten Überlegungen wie Wolfgang-Andreas Schultz' »Melodielehre im Pflichtfach-Unterricht».

Die Sektion zum »Verhältnis von Musiktheorie und Komposition« wurde mit dem anschaulichen Vortrag »Abschaffendes Schaffen: Überlegungen zu einer Poetik kreativer Zerstörung« von Hermann Danuser (Berlin) eröffnet. Diese Sektion war mit siebzehn Vorträgen erwartungsgemäß die größte. Für die vielen ausgesprochen individuellen analytischen Ansätze, die hier sichtbar wurden, ist eine generelle Tendenz charakteristisch: Nur wenige Vorträge thematisierten grundsätzliche Aspekte des Verhältnisses von Musiktheorie und Komposition. Die meisten Beiträge waren analytische Fallstudien, die - und das lag sicher primär am Motto des Kongresses - einzelne musikalische Werke kontextualisierten. Ein Übergewicht eher historisch orientierter Ansätze war deshalb kaum verwunderlich. Stellvertretend seien hier drei unterschiedliche Beiträge erwähnt: Tobias Bleek (Berlin) verfolgte am Beispiel von György Kurtags Webern-Rezeption den unmittelbaren Einfluss von theoretischer Reflektion auf den kompositorischen Schaffensprozess. Astrid Bolay (Trossingen) thematisierte August Halms Analysebegriff vor dem Hintergrund seiner eigenen Kompositionen. Reinhard Bahr (Hamburg) schließlich erörterte mögliche Bezüge zwischen Moritz Hauptmanns dialektisch-spekulativer Musiktheorie und dem Musikdenken Robert Schumanns.

Den Auftakt zur dritten Sektion »Musiktheorie und kultureller Kontext« machte Alexander Rehdings (Harvard) Beitrag »Europäische Musiktheorie und chinesische Musik 1800/1900«, der auf originelle Weise ein in Vergessenheit geratenes Stück früher Musikethnologie thematisierte und dabei die oftmals von zwangsläufigen Missverständnissen geprägte Rezeption außereuropäischer Musikkulturen durch westliche Musik und Musiktheorie aufzeigte. Der historische Zeitraum, der in dieser Sektion durch Beiträge wie »Kulturgeschichtliche Überlegungen zum Modusphänomen in der europäischen Musik« (Tihomir Popovic, Hannover), »Musiktheorie in Zisterzienserklöstern« (Angelika Moths, Basel), „Synchroner Schnitt um 1910: Das kurze Stück im kulturellen Kontext um 1910 « (Simon Obert, Basel) oder auch »Das Geräusch in der Musiktheorie im italienischen Futurismus« (Beate Kutschke, Berlin) abgedeckt wurde, war sehr weit gefasst. Insbesondere Bettina Varwigs (Harvard) Vortrag zu den rhetorischen Grundlagen der Formbildung im 17. Jahrhundert zeigte, wie eine kultur- und geistesgeschichtliche Kontextualisierung - hier besonders der rhetorischen Schriften des Erasmus von Rotterdam - zu einem verblüffend neuen Blick auf das herkömmliche Repertoire beitragen kann. Ein markanter Unterschied zu allen übrigen Sektionen war indes die auffallende Zurückhaltung hinsichtlich der Themen aus dem 20. Jahrhundert. Hier gab es lediglich einen Beitrag von Aaron Girard (Harvard), der sich am Beispiel David Lewins mit den Anfängen der amerikanischen Musiktheorie als intellektueller Disziplin auseinandersetzte. 
Zeichentheoretische und kognitivistische Ansätze spielen in der deutschen Musiktheorie bislang lediglich eine untergeordnete Rolle. Deshalb schien es den Organisatoren angebracht, für diese Sektion drei Eröffnungsvorträge vorzusehen. Dass drei der fünf restlichen Sektionsbeiträge (Uwe Seifert, Martin Pfleiderer und Markus Neuwirth) aus der systematischen Musikwissenschaft kamen, verdeutlicht, dass die deutsche Musiktheorie die «kognitivistische Wende` der amerikanischen Musiktheorie nicht - oder noch nicht - mitvollzieht. Ob die Vorstellung dieser Ansätze auf dem Hamburger Kongress zu einer verstärkten Rezeption in Deutschland führen wird, ist noch nicht abzusehen: Bei aller Offenheit blieb eine gewisse Skepsis spürbar. Neben Christian Thorau, der in das Thema einführte, waren mit Robert Hatten (Bloomington) und Lawrence Zbikowski (Chicago) zwei der renommiertesten Vertreter dieser Strömung nach Hamburg gekommen. Sie näherten sich dem Thema aus jeweils unterschiedlicher Richtung: Hatten gab mit seinem Vortrag »Four Semiotic Approaches to Musical Meaning: Markedness, Topics, Tropes and Gesture einen umfassenden Überblick über seine Arbeiten zur Semiotik. Seine theoretischen Ausführungen illustrierte er mit einer Vielzahl von Musikbeispielen, die die Anwendbarkeit insbesondere der Kategorie der sgesture ‘ auf die musikalische Interpretation aufzeigten. ${ }^{5}$ Zbikowski stellte in seinem Vortrag "Cognitive Science, Music Theory, and Music Analysis « ${ }^{6}$ Analyseverfahren auf der Grundlage von sbasic-level-Kategorien vor. Hier standen anthropologische Grundfragen wie diejenige nach den kognitiven Kapazitäten des Menschen im Vordergrund. Als konkretes Analysebeispiel diente ihm dabei der Film-Song The Way you Look Tonight, den Zbikowski zunächst in seinem originalen filmischen Kontext vorstellte und schließlich auf der E-Gitarre selbstbegleitet darbot. Der Vergleich der beiden Ansätze zeigte die Verlagerung des theoretischen Erkenntnisinteresses - Zbikowski gehört einer jüngeren Generation an als Hatten - von einem primär am konkreten musikalischen Werk orientierten analytischen Zugang hin zu allgemeinen Fragen musikalischer Kognition.

Die Freepaper-Sektion war mit neun Vorträgen nicht sehr umfangreich, dafür inhaltlich aber um so abwechslungsreicher. Martin Schönbergers (München) Beitrag zur Analyse Populärer Musik ${ }^{7}$, der einzige Beitrag seiner Art auf dem Kongress, fand sich in einer Sektion mit Bettina Schergauts (Freiburg) Vortrag zum Verhältnis von musikalischer und dialektischer Logik bei Theodor W. Adorno wieder. Folker Froebe (Mannheim) versuchte in seiner Analyse des I. Satzes von Beethovens Streichquartett op. 95 einen undogmatischen Schenker-Ansatz mit der Tradition motivisch-thematischer Analyse zu verbinden. ${ }^{8}$ Diese Art von Methodeneklektizismus repräsentiert eine wichtige Tendenz in der gegenwärtigen deutschen Musiktheorie, die gerade durch die Verbindung historischer und systematischer Ansätze zu neuen Ergebnissen zu kommen sucht.

Die Sektionen - es fanden bis zu drei Vorträge parallel statt - wurden durch Workshops zu verschiedenen Formen der Improvisation oder auch multimedialer Komposi-

5 Hatten 2004.

6 Ausgehend von seinem 2004 mit dem Wallace-Berry-Award ausgezeichneten Buch Conceptualizing Music (Zbikowski 2002).

7 Siehe den Abdruck des Beitrages in dieser Ausgabe der ZGMTH.

8 Siehe den Abdruck des Beitrages in dieser Ausgabe der ZGMTH. 
tion ergänzt. Präsentationen des Gehörbildungsprogrammes »Orlando« der Dresdner Musikhochschule (John Leigh) sowie zur Zeichensatzanwendung in Funktions- und Stufentheorie ergänzten die theoretischen Vorträge.

Einen weiteren Akzent setzte ein Konzert, in dem Alte und Neue Musik von Machaut bis Ligeti einander gegenübergestellt und mit Texten von Johann Mattheson kommentiert wurde. Schließlich sei auch noch die Podiumsdiskussion zur BA/MA-Studienreform erwähnt, die Fortsetzung einer Veranstaltung vom Vorjahr in Köln, in der die Veränderungen thematisiert wurden, die auf die Musiktheorie als Hochschuldisziplin zukommen werden.

Bei der Abschlussdiskussion gab es zwar wie in den vergangenen Jahren ein überwiegend positives Echo, aber auch kritische Töne wurden laut. Abgesehen von der Kritik an unzureichenden Präsentationsformen einzelner Vortragender wurde ein zu hohes Abstraktionsniveau in vielen Beiträgen kritisiert. Hier handelt es sich aber zweifelsohne um ein Problem, dessen Ursprung nicht nur in der inhaltlichen Gestaltung der Vorträge, sondern in den grundsätzlich divergierenden Ansichten über die Ausrichtung der Musiktheorie, nicht zuletzt der GMTH selbst, zu suchen ist. Für den Verfasser jedenfalls war der Kongress ein beeindruckendes Zeugnis für den Wandel und die zunehmende Professionalisierung der deutschen Musiktheorie. Befürchtungen, das Interesse könnte nach dem euphorischen Beginn in der Gründungsphase der Gesellschaft sukzessiv abnehmen, haben sich nicht bestätigt. Ganz im Gegenteil: Der Kongress hat Vorfreude auf das nächste Treffen der GMTH in Weimar geweckt.

\section{Annual Meeting der SMT in Cambridge/Boston}

Das 28. Annual Meeting der SMT fand vom 10. bis 13. November 2005 im Hyatt Regency-Hotel in Cambridge/Massachussetts, in unmittelbarer Nachbarschaft der Boston University, Harvard und des MIT, statt. Über 500 Teilnehmerinnen und Teilnehmer waren zu diesem Treffen an die amerikanische Ostküste gereist und machten es damit zu dem bisher größten Einzeltreffen der SMT seit ihrer Gründung im Jahr 1978. ${ }^{9}$ Die Zahl der Teilnehmenden, aber auch die Tatsache, dass nahezu jede Hochschule bei den Jahrestreffen der großen akademischen Gesellschaften in den USA präsent ist, macht dieses Treffen nicht nur zu einem wichtigen Forum des akademischen Austausches, sondern auch zum zentralen Academic Job Market: Angesichts der großen geographischen Entfernungen in den USA bietet es sich für die Universitäten, aber auch für die Bewerberinnen und Bewerber an, diese Treffen mit sogenannten `Job Interviews` zu verbinden und so Zeit und Reisekosten zu sparen.

Die programmatische Vielfalt dieses Kongresses lässt jeden Versuch erfolglos erscheinen, die amerikanische Musiktheorie auf bestimmte vorherrschende theoretische Ansätze zu reduzieren. Dennoch lassen sich trotz der zunehmenden Ausdifferenzierung einige Trends beobachten, die im Folgenden dargestellt werden sollen. Im Hinblick auf die in Deutschland momentan stark rezipierte Schenkerian Analysis ist zu sagen, dass dieser

9 Seit einigen Jahren finden die Treffen der SMT alle zwei Jahre gemeinsam mit der American Musicological Society (AMS) statt. 
in den USA ehemals äußerst wichtige Zweig der musikalischen Analyse zwar nach wie vor präsent ist, im Kontext der SMT jedoch kaum noch diskutiert, hinterfragt oder weiterentwickelt wird. In Boston war lediglich eine Session ${ }^{10}$ von insgesamt vierundzwanzig dezidiert der Schenker-Analyse gewidmet: "Schenker: Interruption, Form, and Allusion«. Schenkerian Analysis scheint ihren paradigmatischen Status für die Analyse tonaler Musik in den USA zunehmend einzubüßen. Sie ist auf dem Weg, ein immer noch populäres und an den meisten Universitäten gelehrtes Analyseverfahren zu werden, um das aber keine grundsätzlichen Debatten mehr geführt werden.

Ein neues musiktheoretisches Paradigma stellen demgegenüber die Transformational Theory und die aus ihr hervorgegangene Neo-Riemannian-Theory (NRT) dar. Der große Erfolg dieses theoretischen Ansatzes ist vor allem deswegen erstaunlich, weil sich die NRT nur für einen vergleichsweise kleinen Ausschnitt des (spät-)tonalen Repertoires eignet. In seinem Beitrag für die ZGMTH definiert Edward Gollin die NRT als »a range of speculative and analytical studies concerned with the relation of the mathematical structure of tonal pitch materials (e.g. harmonic triads, tertian seventh chords, diatonic collections, and so forth) to the properties and functions of those materials in musical systems and in individual musical works «. ${ }^{11}$ Der dabei interessanteste Punkt ist Gollins Beschreibung der NRT als »speculative and analytical«. Gollin bezieht sich dabei auf die Anfänge der NRT, die zunächst als spekulatives theoretisches System aus der von David Lewin begründeten Transformational Theory hervorgegangen ist, weshalb einige amerikanische Theoretiker der Ansicht sind, dass die NRT eher Neo-Lewinian-Theory heißen müsste. ${ }^{12}$ Lewins mathematische Überlegungen wurden später von Brian Hyer ${ }^{13}$ und Richard Cohn ${ }^{14}$ aufgegriffen und zur NRT weiterentwickelt. Momentan ist neben der weiteren systematischen Ausdifferenzierung der NRT der Versuch spürbar, die NRT mehr als bisher für die konkrete Analyse fruchtbar zu machen. Gemessen an dem Charakteristikum, zunächst theoretisch-spekulative Modelle zu entwickeln und diese erst in einem zweiten Schritt zum Instrument konkreter Analyse werden zu lassen, kann die NRT als typisch für die amerikanischen Musiktheorie gelten, in der dem spekulativen Anteil musiktheoretischen Denkens traditionell eine größere Rolle zukommt als in der derzeitigen deutschen Musiktheorie.

Lewins entscheidende Rolle bei der Entwicklung der NRT hat ihm in der amerikanischen Musiktheorie das Image des mathematisch und streng systematisch orientierten Theoretikers eingebracht. Dass diese einseitige Bewertung jedoch zu kurz greift, zeigt die posthume Publikation von Lewins beeindruckender Aufsatzsammlung "Studies in Music with Text», die Anfang 2006 als erster Band der neuen Musiktheorie-Reihe bei

10 Es gibt bei den Meetings der SMT zumeist keine thematischen Sektionen, sondern die anonym bewerteten Abstracts werden zu thematisch gebundenen Sessions mit zwei bis vier Vorträgen zusammengefasst.

11 Gollin 2005.

12 Insbesondere Lewins Buch General Musical Intervals and Transformations (1987) ist im Hinblick auf diese Theorieentwicklung paradigmatisch.

13 Hyer 1995.

14 Die wichtigsten Aufsätze in diesem Zusammenhang sind Lewin 1996 sowie Cohn 1996, 1997 und 1998. Für eine umfassende Bibliographie siehe Gollin 2005. 
Oxford University Press ${ }^{15}$ erschienen ist: Der Herausgeber der Reihe, Richard Cohn, bemerkte anlässlich der Präsentation des Bandes auf dem SMT-Kongress, dieses Buch zeige einen »anderen David Lewin«, nämlich einen Theoretiker, der sich fern von jeglichem Systemzwang auf äußerst sensible und erfahrungsnahe Weise der vielschichtigen Analyse von Einzelwerken widme.

In einer Sektion mit dem Titel »Traveling Through Space« wurden eine Reihe systematisch-spekulativer Ansätze aus der Transformational- aber auch der Pitch-Class Set Theory zusammengefasst. Vorträge wie Joseph N. Straus' »Atonal Pitch Space« oder Dmitri Tymoczkos »A Map of All Chords «" ${ }^{16}$ beeindruckten durch ihren Anspruch, Musiktheorie wirklich als \Theorie` im emphatischen Sinne zu begreifen und sich nicht durch Fragen nach der vermeintlichen Relevanz ihres Tuns für die pädagogische Anwendung vorschnell gängeln zu lassen. Andererseits sollten sich - darin mag ein zugegebenermaßen traditionell europäischer Standpunkt zum Ausdruck kommen - gerade die Transformational Theory, die NRT aber auch die Pitch-Class-Set-Theory der Gefahr bewusst sein, dass der Kontakt zur Musik durch ein dominierendes Systemdenken leicht verloren gehen kann. Das Ergebnis können dann Analysen sein, die sich dem hörenden Nachvollzug nahezu vollständig entziehen. Ein Negativbeispiel in dieser Hinsicht war Richard Hermanns (University of New Mexico) Vortrag »Parsimonious Equivalence Classes for Voice-Leading between Maximally Even and Near Maximally Even Set Classes«, der schon in seiner Titelgebung die hier gemeinte Problematik widerspiegelt.

Vor diesem Hintergrund - der Diskrepanz zwischen analytischen Ergebnissen und ihrem Beitrag für die Konstituierung des ästhetischen Gegenstandes Musik - ist auch das zunehmende Interesse in den USA an einer Verbindung von Musiktheorie und Kognition zu verstehen: Neben traditionell musikpsychologisch orientierten Vorträgen wie »Surprise and Listening Ahead: Analytic Engagements with Musical Tendencies" von Elizabeth Hellmuth Margulis (Northwestern University) griff Clifton Callender (Florida State University) in seinem Vortrag "Some thoughts on Measuring Voice-Leading Distance» die für die NRT zentrale Kategorie des 'parsimonious voice-leading ‘ auf und stellte grundlegende theoretische Erkenntnisse der NRT hinsichtlich ihrer musikalischen Wahrnehmbarkeit durch eine empirische Untersuchung auf den Prüfstein. ${ }^{17}$ Neben den bisher beschriebenen Entwicklungen ist - ähnlich wie in Deutschland - eine Tendenz zur eklektischen Kombination unterschiedlicher methodischer Ansätze zu beobachten. Deutlich wurde dies insbesondere in der Session »Combining Musical Systems« bei Jonathan R. Pieslaks (University of Michigan) Vortrag »Three Turns sin the Evening Air : Schenkerian, Schoenbergian, and Neo-Riemannian Perspectives on a Debussy-Prelude».

16 Eine aktuelle Version von Tymoczkos SMT-Vortrag ist kürzlich unter dem Titel »The Geometry of Musical Chords « in Science veröffentlich worden (Science, 7. Juli 2006, Vol. 313, Nr. 5783, 72-74). Es ist damit der erste Musik-Artikel in der über 120-jährigen Geschichte der wichtigsten naturwissenschaftlichen Zeitschrift der USA und steht für die naturwissenschaftlich-systematische Ausrichtung von Teilen der >Music Theory` in den Vereinigten Staaten. Der Text und zusätzliches Material sind auf Tymoczkos Homepage frei zugänglich (http://www.music.princeton.edu/ dmitri/sciencearticle.htm, 12.6.2006).

Siehe in diesem Kontext auch Callender 1998. 
Einer der interessantesten Vorträge war lan Quinns »Harmonic Function without Primary Triads", der in der Session »Tonal Reconstructions" angesiedelt war. Quinn, derzeit Assistant Professor an der Yale University, berichtete über einige von ihm eingeführte Neuerungen im Undergraduate-Curriculum. Im Mittelpunkt steht dabei eine AkkordChiffrierung, die Quinn sfunctional-bass notation nennt, eine Kombination aus Funktionssymbolen und Generalbassziffern, die eine Abkehr vom Umkehrungsdenken der in den USA durch sroman numerals« repräsentierten Stufenlehre bedeutet und dabei unter anderem einen bereits von Daniel Harrison skizzierten Funktionsbegriff weiterentwickelt. ${ }^{18}$ Diese Reform des Theorieunterrichts zielt auf die schnellere individuelle Arbeit der Studenten am konkreten musikalischen Material, in erster Linie dem Erstellen von Stilkopien. Bemerkenswert an Quinns Ansatz ist neben den konkreten Ideen zur Umsetzung im Unterricht, dass er diesen Innovationen einen theoretisch sehr komplex begründeten Tonalitätsbegriff zugrunde legt, für den er auch empirische Ergebnisse aus der Kognitionsforschung heranzieht. ${ }^{19}$ Quinns Vortrag war daher eine anregende Verknüpfung von Erfahrungen aus einer propädeutischen Lehrsituation und abstrakt-spekulativer Forschung. Konkrete Unterrichtspraxis und spekulative Theoriebildung werden in den USA - und das gilt nicht nur für Quinns Vortrag - nicht als unüberbrückbarer Gegensatz, sondern zumeist als sich gegenseitig befruchtend angesehen. ${ }^{20}$ Gerade in diesem Bereich zeigen sich jedoch Unterschiede zwischen der Situation in den USA und Deutschland: Während neue Ansätze im Bereich des Satzlehreunterrichts hierzulande in letzter Zeit zunehmend historisch ausgerichtet sind, werden die umfangreichen Forschungen zur Geschichte der Musiktheorie in den USA in der Regel nicht für die Herausbildung neuer theoretischer Ansätze fruchtbar gemacht.

Eine ganz andere Art, musiktheoretische Erkenntnisse in der pädagogischen Praxis anzuwenden, zeigte sich im Bereich »Performance and Analysis«. In einer Sektion zu »Performance and Rhythm« wurden Forschungsergebnisse zu »Chopin's Chromatic Shadings: Performance as Analysis« oder »Glenn Gould's Constant Rhythmic Reference Point « vorgestellt. Einer der inhaltlichen Höhepunkte des SMT-Treffens war jedoch die Abendsektion »Interacting Interpretive Roles - Performer and Theorist", in der drei Theoretiker Studierende des New England Conservatory in Boston live gecoacht haben. Clemens Kemme (Conservatorium van Amsterdam) betonte im Lied /l pleure dans mon cœur von Debussy aufführungsrelevante Zusammenhänge zwischen Text und Musik. William Rothstein (CUNY) legte bei seinem Unterricht von Brahms' 2. Violinsonate op. 100 den Schwerpunkt auf strukturell begründbare interpretatorische Alternativversionen. Robert Hatten machte schließlich seinen - bereits auf dem Hamburger GMTHKongress vorgestellten - gestischen und rhetorischen Ansatz für die Interpretation des III. Satzes von Beethovens Streichquartett B-Dur, op. 130 fruchtbar. Kemmes Textinter-

18 Harrison 1994.

19 Quinns Ph. D. Dissertation A Unified Theory of Chord Quality in Equal Temperaments (2004) widmet sich einer ähnlichen Thematik und erscheint sukzessive in drei Abschnitten in Perspectives of New Music.

20 Dass auf den Treffen der SMT relativ wenig dezidiert pädagogische Themen diskutiert werden, ist darauf zurückzuführen, dass diese Themen tendenziell bei den ebenfalls jährlich stattfindenden Treffen der College Music Society (http://www.music.org) behandelt werden, der mit fast 10.000 Mitgliedern größten akademischen Musikgesellschaft der USA. 
pretation, Rothsteins struktureller Zugang und Hattens gestische Interpretation sind drei sehr unterschiedliche Ansätze, die jeder auf seine Art ein großes Potential für ein gewinnbringendes Zusammenwirken von Theorie und musikalischer Interpretation bergen. Das überwältigende Publikumsinteresse an dieser Veranstaltung zeigte die Bedeutung, die diesem bisher sicherlich zu wenig beachteten Thema in Zukunft innerhalb der Musiktheorie zukommen wird.

Die thematische Ausrichtung der Bela Bartóks drittem Streichquartett gewidmeten Plenary Session - unmittelbar vor dem Business-Meeting der SMT - steht beispielhaft für das anhaltend große Interesse der amerikanischen Musiktheorie an der Musik des 20. Jahrhunderts. Dabei scheint Bartók die bisher dominierenden Repräsentanten der musikalischen Moderne, Schönberg und Strawinsky, zunehmend abzulösen. Die drei analytischen Vorträge von Joseph N. Straus (CUNY), Jonathan W. Bernard (University of Washington) und Judith Frigyesi (Bar-Ilan University) wurden durch eine Aufführung des Streichquartetts durch das Borromeo String Quartet aus Boston ergänzt. Daneben gab es noch eine Reihe weiterer Sessions zur Musik des 20. Jahrhunderts mit Themen wie »American Composers since 1945«, »Schoenberg and Leibowitz« und »European Twentieth-Century Composers".

Auch die Publication Awards der SMT im Jahr 200521 reflektieren die bisher beschriebenen Entwicklungen innerhalb der amerikanischen Musiktheorie. Den Preis für den besten Artikel erhielt Michael Klein (Temple University) für seinen Aufsatz »Chopin's Fourth Ballade as Musical Narrative«. Klein kombiniert dort musiktheoretische, musikwissenschaftliche, semiotische und hermeneutische Ansätze zu einer eigenständigen und in ihrer methodischen Bandbreite exemplarischen musikalischen Analyse, in deren Mittelpunkt das Konzept der Intertextualität steht. Der Emerging Scholar Award ging an Julian Hook (Indiana University) für seinen Artikel "Uniform Triadic Transformations", die produktive Weiterentwicklung und Systematisierung einer Reihe von Ideen der Transformational Theory. Der wichtigste Preis, den die SMT zu vergeben hat, der WallaceBerry-Award für das beste Buch, wurde dem Ethnomusicologist Marc Perlman (Brown University) für Unplayed Melodies: Javanese Gamelan and the Genesis of Music Theory verliehen. Perlman überwindet in diesem Buch die Grenzen des Faches durch die Verbindung traditioneller westlicher Musiktheorie mit der Ethnomusicology und den Kognitionswissenschaften. Der in den USA - anders als in Deutschland - primär innerhalb der Disziplin Musiktheorie selbst angesiedelte Bereich der Geschichte der Musiktheorie wurde durch zwei Special Citations berücksichtigt. Zum einen wurde Claude Paliscas Übersetzung und Kommentar von Vincenzo Galileis Dialogo della musica antica et della moderna (Florenz 1581) ausgezeichnet, zum anderen eine zweibändige Übersetzung von Heinrich Schenkers Der Tonwille - ein großes Projekt unter der Editionsleitung von William Drabkin.

21 Ausführliche Beschreibungen der einzelnen Publikationen und vollständige bibliographische Angaben unter: http://www.societymusictheory.org/index.php?pid=187 (12.6.2006). 
Fazit

Das Jahrestreffen der SMT 2005 hat erneut die große Leistungsfähigkeit und Vielseitigkeit der amerikanischen Musiktheorie unter Beweis gestellt. Was bedeutet das für die deutsche Musiktheorie? Deren Potential liegt immer noch in der sehr soliden handwerklichen Ausbildung an den Musikhochschulen und der engen Verbindung zur künstlerischen Praxis. Die Gründung der GMTH hat der bisher tendenziell vernachlässigten wissenschaftlichen Seite des Faches ein stärkeres Gewicht verliehen. Bei der weiteren Entwicklung eines eigenständigen Profils kann der Dialog mit der amerikanischen Musiktheorie hilfreich sein, wenn die Vielfalt des amerikanischen Diskurses fruchtbar gemacht wird, ohne darauf zu verzichten, bestimmte Entwicklungen kritisch zu hinterfragen.

Sehr auffällig ist, dass die Ansätze, die momentan die Diskussion in den USA beherrschen, wie beispielsweise die NRT oder die kognitive Musiktheorie, in der deutschen Musiktheorie nur eine marginale Rolle spielen. Doch ist diese Skepsis gegenüber unhistorisch-systematischen Entwürfen, denen der Versuch eines innovativen Umgangs mit historischen Erkenntnissen gegenübergestellt wird, vielleicht bereits der Anfang einer eigenständigen Profilbildung der deutschen Musiktheorie im Vergleich zu einer immer noch stark systematisch orientierten Musiktheorie in den USA, eine Orientierung, die sich durch die NRT derzeit wieder zu verstärken scheint.

Der deutlichste Unterschied zwischen amerikanischer und deutscher Musiktheorie betrifft aber möglicherweise gar keinen inhaltlichen Aspekt, sondern die Kommunikationsstruktur innerhalb der sScientific Community،. Auf Grund der Tatsache, dass sich die Musiktheorie in Deutschland erst vor wenigen Jahren in Form der GMTH einen institutionellen Rahmen gegeben hat, sind wechselseitige Bezugnahmen auf Entwicklungen innerhalb des Faches immer noch rar. Auch der Hamburger Kongress vermittelte den Eindruck, dass unterschiedliche Ansätze, nachdem sie vorgestellt worden sind, doch zumeist unverbunden nebeneinander stehen bleiben. Daher wären in Zukunft offenere und offensiv geführte inhaltliche Diskussionen wünschenswert. Ob die jährlichen Kongresse dafür ein angemessenes Forum bilden können, scheint jedoch nicht nur aus organisatorischen Gründen fraglich. Eine Möglichkeit könnten zwar inhaltlich-programmatische Podiumsdiskussionen bieten, die auf den bisherigen Kongressen überwiegend zu institutionellen Fragen durchgeführt wurden ${ }^{22}$, eine aussichtsreichere andere Option könnte aber darin bestehen, rregionale` Treffen zu spezifischen Themenstellungen dem Jahreskongress flankierend an die Seite zu stellen, so wie dies zuletzt mit den Symposien zum Schaffen von Sethus Calvisius in Leipzig und zum Thema »Musikalische Logik und musikalischer Zusammenhang" in Bremen bereits geschehen ist. Auch hier bedarf es allerdings, nicht anders als bei den Jahreskongressen, jeweils der Klärung, wie die Zusammenarbeit der GMTH mit den musiktheoretischen Fachbereichen der einzelnen Hochschulen aussehen könnte.

Jan Philipp Sprick

22 Dass auch institutionelle Diskussionen inhaltliche Fragen berühren, hat die BA/MA-Diskussion auf dem Hamburger Kongress gezeigt, da die Positionierung der Musiktheorie in der Neuordnung der Hochschulen zentrale Fragen des fachlichen Selbstverständnisses berührt. 


\section{Literatur}

Callender, Clifton (1998), „Voice-Leading Parsimony in the Music of Alexander Scriabin«, Journal of Music Theory 42, 219-233.

Cohn, Richard (1996), »Maximally Smooth Cycles, Hexatonic Systems, and the Analysis of Late-Romantic Triadic Progressions", Music Analysis 15, 9-40.

(1997), „Neo-Riemannian Operations, Parsimonious Trichords, and Their Tonnetz Representations", Journal of Music Theory 41, 1-66.

— (1998), »Introduction to Neo-Riemannian Theory: A Survey and Historical Perspective«, Journal of Music Theory 42, 167-180.

Gollin, Edward (2005), »Neo-Riemannian Theory«, Zeitschrift der Gesellschaft für Musiktheorie 1-2 (2003/05), Bd. 2: 2/2-3, 153-155.

Harrison, Daniel (1994), Harmonic Function in Chromatic Music, Chicago.

Hatten, Robert (2004), Interpreting Musical Gestures, Topics, and Tropes: Mozart, Beethoven, Schubert, Bloomington: Indiana University Press.

Hyer, Brian (1995), »Reimag(in)ing Riemann«, Journal of Music Theory 39, 101-138.

Lewin, David (1987), Generalized Musical Intervals and Transformations, New Haven: Yale University Press.

_ (1996), „Cohn Functions«, Journal of Music Theory 40, 181-216. (2006), Studies in Music with Text, Oxford: Oxford University Press.

Quinn, Ian (2004), A Unified Theory of Chord Quality in Equal Temperaments, Ph. D. Diss., Eastman School of Music.

Zbikowski, Lawrence M. (2002), Conceptualizing Music. Cognitive Structure, Theory, and Analysis, Oxford: Oxford University Press. 